\title{
ON ALMOST ADDITIVE FUNCTIONS
}

\section{JANUSZ BRZDȨK}

Let $(S,+)$ be a semigroup and $(H,+)$ be a group (neither necessarily commutative). Suppose that $J \subset 2^{S}$ is a proper ideal in $S$ such that

$$
\begin{gathered}
x+A \in J \text { for } A \in J, x \in S, \\
x+A, A+x \notin J \text { for } A \in 2^{S} \backslash J, x \in S,
\end{gathered}
$$

and $\Omega(J)=\left\{M \subset S^{2}:\right.$ there exists $U(M) \in J$ with $M[x] \in J$ for $\left.x \in S \backslash U(M)\right\}$, where $M[x]=\{y \in S:(y, x) \in M\}$. We show that if $f: S \rightarrow H$ is a function satisfying

$$
f(x+y)=f(x)+f(y) \quad \Omega(J) \text {-almost everywhere in } S^{2},
$$

then there exists exactly one additive function $F: S \rightarrow H$ with $F(x)=f(x)$ $J$-almost everywhere in $S$.

We also prove some results concerning regularity of the function $F$.

Motivated by a problem raised by Erdös [4], several authors studied almost additive functions (see $[2,6,7,8,9,10]$ ), that is functions satisfying the Cauchy equation

$$
f(x+y)=f(x)+f(y)
$$

almost everywhere (the notion "almost everywhere" is explained in the sequel). In particular, Ger [6] proved the following abstract theorem.

Theorem A. (See [6, Theorem 1].) Let $(G,+),(H,+)$ be two groups (not necessarily commutative) and let $J$ be a proper linearly invariant ideal in $G$. Suppose that $S$ is a subsemigroup of $G$ with $S \notin J$ and

$$
S-S:=\{x-y: x, y \in S\}=G
$$

and $f: S \rightarrow H$ satisfies

$$
f(x+y)=f(x)+f(y) \quad \Omega(J) \text {-almost everywhere in } S^{2} .
$$

Received 8 November 1995

Copyright Clearance Centre, Inc. Serial-fee code: 0004-9729/96 \$A2.00+0.00. 
Then there is exactly one additive function $F: G \rightarrow H$ with $F(x)=f(x) J$-almost everywhere in $S$.

Let us recall (see [6] and [7]) that $J \subset 2^{G}$ is a proper ideal in $G$ provided the following three conditions are valid:

$$
\begin{gathered}
G \notin J, \\
A \cup B \in J \text { for } A, B \in J, \\
\text { if } A \in J \text { and } B \subset A, \text { then } B \in J .
\end{gathered}
$$

Further, $J$ is linearly invariant if

$$
\{x-A: x \in G\} \subset J \text { for every } A \in J
$$

where $x-A=\{x-z: z \in A\}$. Finally

$$
\Omega(J)=\left\{M \subset G^{2}: \text { there is } U(M) \in J \text { with } M[x] \in J \text { for } x \in G \backslash U(M)\right\},
$$

where $M[x]=\{y \in G:(y, x) \in M\}$. (The condition defining $\Omega(J)$ is an abstract equivalent of the Fubini Theorem.)

We remind the reader of the notion "almost everywhere". Given an ideal $J$ in $G\left(G^{2}\right.$, respectively), we say that a property $P(x), x \in D \subset G\left(x \in D \subset G^{2}\right.$, respectively), holds $J$-almost everywhere in $D$ provided there is $A \in J$ such that $P(x)$ holds for every $x \in D \backslash A$.

We prove a generalisation of Theorem A; in particular, we show that assumption (1) is not necessary and we can confine ourselves to ideals in $S$. Moreover, our proof is shorter. We give also some results concerning the regularity of the function $F$.

Some examples of the spaces satisfying the abstract hypotheses, which are used in the sequel, are given at the end of the paper.

Since we are going to consider only ideals in $S$, we must replace (3) by an assumption expressed in the terms of $S$. We shall use the following two conditions:

$$
\begin{aligned}
x+A \in J & \text { for } A \in J, x \in S, \\
x+A, A+x \notin J & \text { for } A \in 2^{S} \backslash J, x \in S .
\end{aligned}
$$

Note that if $S$ is a group, then (4) and (5) are equivalent to the following condition:

$$
x+A+y \in J \quad \text { for } A \in J, x, y \in S .
$$

It is easily seen that if $S$ is a subsemigroup of a group $(G,+)$ and $J \subset 2^{G}$ satisfies (3), then (4) and (5) hold (compare [7, Remark]). Furthermore, condition (3) is stronger than (4) and (5), because it also yields

$$
-A \in J \text { for } A \in J \text {. }
$$


Given $J \subset 2^{S}$ we define $\Omega(J)$ in the same way as before (with $G=S$ ).

Now, we are in a position to formulate our main result. Namely, we have the following theorem.

THEOREM 1. Let $(S,+)$ be a semigroup and $(H,+)$ be a group (neither necessarily commutative). Suppose that $J \subset 2^{S}$ is a proper ideal in $S$ such that (4) and (5) hold and $f: S \rightarrow H$ is a function satisfying (2). Then there exists exactly one additive function $F: S \rightarrow H$ with $F(x)=f(x) J$-almost everywhere in $S$.

For the proof we need the following lemma.

Lemma 1. Let $A \in J$ and $S_{0}=S \backslash A$. Then

$$
d\left(S_{0}\right):=\left\{x \in S:\left(x+S_{0}\right) \cap S_{0} \neq \emptyset\right\}=S .
$$

Proof: It suffices to show that $S \subset d\left(S_{0}\right)$. Fix $y \in S$. Then (4) implies

$$
\begin{aligned}
&(y+S) \backslash\left(S_{0} \cap\left(y+S_{0}\right)\right) \\
&= {\left[A \cap(y+S) \cup S_{0} \cap(y+S)\right] \backslash\left[S_{0} \cap\left(y+S_{0}\right)\right] \subset A \cup(y+A) \in J . }
\end{aligned}
$$

Since, by (5), $y+S \notin J$, this means that $S_{0} \cap\left(y+S_{0}\right) \neq \emptyset$. Hence $y \in d\left(S_{0}\right)$.

Proof of Theorem 1: Given $D \subset S$ and $s \in S$, in the sequel, we denote $D^{\mathbf{s}}=\{t \in S: t+s \in D\}$ and $D_{s}=\{t \in S: s+t \in D\}$.

According to the hypotheses there are $M \in \Omega(J)$ and $U(M) \in J$ such that

$$
f(x+y)=f(x)+f(y) \quad \text { for }(x, y) \in S^{2} \backslash M
$$

and $M[x] \in J$ for every $x \in S \backslash U(M)$. First we prove that

(6) $f(x)-f(y)=f(u)-f(v)$ for $x, y, u, v \in S \backslash U(M), z \in S$ with $x=z+y$ and $u=z+v$.

Fix $x, y, u, v \in S \backslash U(M)$ and $z \in S$ such that $x=z+y$ and $u=z+v$. Observe that, by (5), $M[y]^{z}, M[v]^{z} \in J$. Consequently there is

$$
s \in S \backslash\left(M[x] \cup M[u] \cup M[y]^{z} \cup M[v]^{z}\right) .
$$

It is easily seen that $(s, x),(s, u),(s+z, y),(s+z, v) \notin M$. Thus

$$
\begin{aligned}
& f(s)+f(x)=f(s+x)=f(s+z+y)=f(s+z)+f(y), \\
& f(s)+f(u)=f(s+u)=f(s+z+v)=f(s+z)+f(v) .
\end{aligned}
$$

Hence $-f(u)+f(x)=-f(v)+f(y)$. This implies (6). 
Now, on account of Lemma 1 and (6), we can define a function $F: S \rightarrow H$ by the formula:

$$
F(z)=f(x)-f(y) \text { for } z \in S,
$$

where $x$ and $y$ are any elements of $S \backslash U(M)$ such that $x=z+y$. We shall show that $F$ is additive and $F(x)=f(x) J$-almost everywhere in $S$.

To this end, fix $z, w \in S$. Take $y \in S$ and set $x=z+y$ and $u=w+x$. In view of (5), $U(M)_{x}, U(M)_{y}, U(M)_{u} \in J$, which means that there is $t \in S \backslash$ $\left(U(M)_{x} \cup U(M)_{y} \cup U(M)_{u}\right)$. Put $x_{t}=x+t, y_{t}=y+t$, and $u_{t}=u+t$. Then $x_{t}, y_{t}, y_{t} \notin U(M), x_{t}=z+y_{t}, u_{t}=w+x_{t}$, and $u_{t}=w+x_{t}=w+z+y_{t}$. Hence, according to the definition of $F$,

$$
F(w)+F(z)=f\left(u_{t}\right)-f\left(x_{t}\right)+f\left(x_{t}\right)-f\left(y_{t}\right)=f\left(u_{t}\right)-f\left(y_{t}\right)=F(w+z) .
$$

Take $y \in S \backslash U(M)$. Then $A:=U(M)^{y} \cup M[y] \in J$ and, for every $x \in S \backslash A$, $(x, y) \notin M$ and $x+y \notin U(M)$. This implies that

$$
F(x)=f(x+y)-f(y)=f(x)+f(y)-f(y)=f(x) \text { for } x \in S \backslash A .
$$

To complete the proof suppose that $H: S \rightarrow G$ is also an additive function such that $H(x)=f(x)$ for $x \in S \backslash D$ with some $D \in J$. Then $B=A \cup D \in J$ and

$$
F(x)=H(x) \text { for } x \in S \backslash B \text {. }
$$

Fix $z \in S$. In view of Lemma 1 , there are $x, y \in S \backslash B$ with $x=z+y$. Thus

$$
F(z)=F(z+y)-F(y)=F(x)-F(y)=H(x)-H(y)=H(z) .
$$

This ends the proof.

REMARK 1. Theorem A can be easily derived from Theorem 1 . In fact, let $G, H, S$, $f$ and $J$ be just the same as in Theorem A. Then $J_{S}:=J \cap 2^{S}=\{A \cap S: A \in J\}$ is a proper ideal in $S$ and (4), (5) are valid with $J$ replaced by $J_{S}$. Put $M:=\{(x, y) \in$ $\left.S^{2}: f(x+y) \neq f(x)+f(y)\right\}$ and $U(M):=\{x \in S: M[x] \notin J\}=\left\{x \in S: M[x] \notin J_{S}\right\}$. Note, that, according to the hypotheses on $f, U(M) \in J_{S}$ and $M[x] \in J_{S}$ for every $x \in S \backslash U(M)$. Thus, in view of Theorem 1 , there is a unique additive function $F_{S}$ mapping $S$ into $H$ with $f(x)=F_{S}(x) J_{S}$-almost everywhere in $S$. Further, according to [1, Theorem 3], $F_{S}$ can be extended to an additive function $F: G \rightarrow H$ in a unique way. Since $J_{S} \subset J$, we have $f(x)=F(x) J$-almost everywhere in $S$.

Now, we are going to prove some results concerning the regularity of the function $F$. In the sequel, given a semigroup $(S,+)$ and $x \in S$, we denote by $L_{x}, R_{x}$ the functions mapping $S$ into $S$ and defined by:

$$
L_{x}(y)=x+y, R_{x}(y)=y+x \text { for } y \in S
$$


Let us begin with an abstract case of the well known Steinhaus Theorem (see [11] and [12]).

Proposition 1. Let $(S,+)$ be a semigroup (not necessarily commutative) with zero, endowed with a topology such that

$$
L_{x} \text { and } R_{x} \text { are continuous at } 0 \text { for every } x \in S \text {. }
$$

Suppose that $J \subset 2^{S}$ is an ideal in $S$ satisfying (4) and such that

$$
y+W \notin J \text { for every } y \in S \text { and every neighbourhood } W \subset S \text { of } 0 .
$$

Then, for every non-empty open set $U \subset S$ and every $A \in J, 0 \in \operatorname{int} d(U \backslash A)$.

Proof: Fix a non-empty open set $U \subset S$ and $A \in J$. Take $x \in U$ and put $U_{0}=U \backslash A$. According to (7), there is an open neighbourhood $V \subset S$ of 0 with $V+x \subset U$. It suffices to show that $V \subset d\left(U_{0}\right)$.

To this end, fix $y \in V$. It is easily seen that $y+x \in U \cap(y+U)$. Whence and on account of continuity of $L_{y+x}$ and $L_{x}$ at 0 , there is a neighbourhood $W \subset S$ of 0 with $y+x+W \subset U$ and $x+W \subset U$, and consequently $y+x+W \subset U \cap(y+U)$. Since

$$
[U \cap(y+U)] \backslash\left[U_{0} \cap\left(y+U_{0}\right)\right] \subset A \cup(y+A) \in J,
$$

(8) implies that $U_{0} \cap\left(y+U_{0}\right) \neq \emptyset$, which means that $y \in d\left(U_{0}\right)$. This completes the proof.

Remark 2. Lemma 1 can be easily derived from Proposition 1 ; it suffices only to assume the trivial topology in $S$ (that is such that $S$ and $\emptyset$ are the only open sets). However, since the proof of Lemma 1 is very short and simple, for the convenience of the reader we presented it separately.

ThEOREM 2. Let $S, H, J$ and $f$ be just the same as in Theorem 1. Moreover, assume that $H$ is a topological group, $0 \in S, S$ is endowed with a topology such that (7) and ( 8 ) hold, and $f$ is continuous at a point $x_{0} \in S$. Then there exists an additive function $F: S \rightarrow H$, continuous at 0 , such that $f(x)=F(x) J$-almost everywhere in $S$.

Proof: According to Theorem 1, there are an additive function $F: S \rightarrow H$ and $A \in J$ such that $f(x)=F(x)$ for every $x \in S \backslash A$. We show that $F$ is continuous at 0 .

By the additivity of $F$ we have $F(0)=0$. Fix a neighbourhood $V \subset H$ of 0 . There are an open neighbourhood $U \subset S$ of $x_{0}$ and a neighbourhood $W \subset H$ of 0 such that $W-W \subset V$ and $f(U) \subset W+f\left(x_{0}\right)$. Let $U_{0}=d(U \backslash A)$. Then

$$
F\left(U_{0}\right) \subset F(U \backslash A)-F(U \backslash A) \subset f(U)-f(U) \subset W-W \subset V .
$$

Since $U_{0}$ is a neighbourhood of 0 from Proposition 1, this ends the proof. 
REMARK 3. It is easy to show that if $(S,+)$ is a group endowed with a topology such that the translation $L_{x}$ is continuous for every $x \in S$, then every additive function $F$ continuous at 0 mapping $S$ into a topological group is continuous. This is not the case when $S$ is "only" a semigroup. The following example shows this.

EXAMPLE. Let $Q^{+}$stand for the set of all non-negative rationals and let $b \notin Q^{+}$be a positive real number. Put

$$
S=\left\{p+b q: p, q \in Q^{+}\right\}
$$

and

$$
F(p+b q)=p \text { for every } p, q \in Q^{+} .
$$

Then $F: S \rightarrow Q$ ( $Q$ denotes the set of all rationals) is additive and, if we assume in $S$ and $Q$ the topologies induced from the real line, it is continuous at 0 and discontinuous at every point of $S \backslash\{0\}$.

However, in numerous other cases (for example when $S=[0,+\infty$ ) with the usual topology) every additive function continuous at 0 mapping $S$ into a topological group is continuous.

Now, we shall study the case where $f$ is measurable. Given a semigroup $(S,+)$ endowed with a topology and $T, J \subset 2^{S}$, we shall use the following hypotheses:

$$
\begin{gathered}
B \backslash A \in T \text { for } B \in T, A \in J, \\
0 \in \operatorname{int} d(B) \text { for } B \in T \backslash J, \\
2 A:=\{2 x: x \in A\} \in J \text { for } A \in J .
\end{gathered}
$$

We need also the following three definitions.

Definition 1: Let $S \neq \emptyset$ be a set and $J \subset 2^{S}$. We say that $J$ is a proper $\sigma$-ideal in $S$ provided it is a proper ideal in $S$ and

$$
\bigcup\left\{A_{n}: n \in N\right\} \in J \text { for }\left\{A_{n}: n \in N\right\} \subset J,
$$

where $N$ denotes the set of all positive integers.

Definition 2: Let $S$ and $H$ be topological spaces and $T \subset 2^{S}$. We say that a function $f: S \rightarrow H$ is $T$-measurable provided $f^{1}(U) \in T$ for every open set $U \subset H$.

Definition 3: (See [3].) A topological group $(H,+)$ is $\sigma$-bounded provided, for every neighbourhood $U \subset H$ of 0 , there is a sequence $\left(x_{n}: n \in N\right) \subset H$ with

$$
H=\bigcup\left\{U+x_{n}: n \in N\right\} .
$$

For instance, if a topological group has a countable dense subset, then it is $\sigma$ bounded.

We have the following. 
Theorem 3. Let $S, H, J$ and $f$ be just the same as in Theorem 1. Moreover, assume that $B$ is a topological group, $0 \in S, T$ is a family of subsets of $S$ satisfying (9) and (10), $J$ is a $\sigma$-ideal, $f$ is $T$-measurable, and one of the following two conditions holds:

(i) $H$ is $\sigma$-bounded,

(ii) $S$ is divisible by 2 (that is, for every $x \in S$ there is $z \in S$ with $x=2 z$ ), (11) holds, $2 x \neq 2 y$, for every $x, y \in H$ with $x \neq y$, and for every neighbourhood $U \subset \mathrm{H}$ of 0

$$
H=\bigcup\left\{2^{n} U: n \in N\right\}
$$

Then there is an additive function $F: S \rightarrow H$, continuous at 0 , such that

$$
f(x)=F(x) \quad J \text {-almost everywhere in } S .
$$

Proof: In view of Theorem 1, there are an additive function $F: S \rightarrow H$ and $A \in J$ such that $F(x)=f(x)$ for $x \in S \backslash A$. We show that $F$ is continuous at 0 . To this end, fix open neighbourhoods $U, V \subset H$ of 0 with

$$
V-V \subset U \text {. }
$$

First, consider the case where (i) holds. Then there is a sequence $\left\{x_{n}: n \in N\right\} \subset H$ such that

$$
H=\bigcup\left\{V+x_{n}: n \in N\right\}
$$

Since $S \notin J$ and

$$
S=f^{-1}(H)=\bigcup\left\{f^{-1}\left(V+x_{n}\right): n \in N\right\},
$$

there exists $k \in N$ with

$$
S_{k}:=f^{-1}\left(V+x_{k}\right) \notin J .
$$

Hence, by (9), $B:=S_{k} \backslash A \in T \backslash J$. Further, in view of (10), $0 \in \operatorname{int} d(B)$ and

$$
F(d(B)) \subset F(B)-F(B) \subset f\left(S_{k}\right)-f\left(S_{k}\right) \subset V-V \subset U .
$$

Now, suppose that (ii) is satisfied. Then

$$
S=f^{-1}(H)=f^{-1}\left(\bigcup\left\{2^{n} V: n \in N\right\}\right)=\bigcup\left\{f^{-1}\left(2^{n} V\right): n \in N\right\} .
$$

Thus there exists $k \in N$ with

$$
S_{k}:=f^{-1}\left(2^{k} V\right) \notin J
$$


Since, by (11), $2^{k} A:=\left\{2^{k} x: x \in A\right\} \in J$, we have also $S_{k} \backslash\left(A \cup 2^{k} A\right) \notin J$.

Take $y \in S_{k} \backslash\left(A \cup 2^{k} A\right)$. Then $f(y)=2^{k} f(x)$ for some $x \in f^{-1}(V)$ and, according to the hypotheses on $S$, there is $z \in S$ such that $y=2^{k} z$. Note that $z \notin A$, because $y \notin 2^{k} A$. Thus

$$
2^{k} f(x)=f(y)=F(y)=F\left(2^{k} z\right)=2^{k} F(z)=2^{k} f(z),
$$

which means that $f(z)=f(x) \in V$ and consequently

$$
y=2^{k} z \in 2^{k} f^{-1}(V)
$$

In this way we have shown that

$$
S_{k} \backslash\left(A \cup 2^{k} A\right) \subset 2^{k} f^{-1}(V) .
$$

Thus $2^{k} f^{-1}(V) \notin J$ and, by virtue of (9) and (11),

$$
B:=f^{-1}(V) \backslash A \in T \backslash J .
$$

Hence, on account of (10), $0 \in \operatorname{int} d(B)$. To complete the proof it suffices to note that

$$
F(d(B)) \subset F(B)-F(B) \subset f(B)-f(B) \subset V-V \subset U .
$$

Let us observe that the assumptions of (ii) which concern $H$ are satisfied in every real topological linear space. Below, we present some examples of families $T$ and proper $\sigma$-ideals $J$ which satisfy (9) and (10).

1. $S$ is a locally compact topological group, $T \subset 2^{S}$ is the $\sigma$-algebra of all Haar measurable sets, and $J$ is the $\sigma$-ideal of all subsets of $S$ of Haar measure zero (see [12]).

2. $S$ is a Polish commutative group, $T \subset 2^{S}$ is the $\sigma$-algebra of all Christensen measurable sets, and $J$ is the $\sigma$-ideal of all Christensen zero subsets of $S$ (see [5]; compare also [8]).

3. $S$ is a semitopological group such that every non-empty open set is of the second Baire category in $S, T \subset 2^{S}$ is the $\sigma$-algebra of all sets with the Baire property, and $J$ is the $\sigma$-ideal of the first category subsets of $S$ (see [11] and Proposition 1).

4. $S$ is a semigroup with 0 , endowed with a topology such that (7) holds, $J \subset 2^{S}$ is the $\sigma$-ideal satisfying (8), and $T=\{(U \backslash A) \cup B: U \subset$ $S$ is open and $A, B \in J\}$ (see Proposition 1). 
Moreover, the ideals in examples 1-3 also satisfy (4), (5) and (8) and, if $S$ is a topological linear space, then they also satisfy (11).

For a discussion on $\Omega(J)$ for $J$ described in Example 2 we refer to [8].

Simple proper ideals $J$ such (4) and (5) are valid are provided by the family of all bounded sets and the family of all sets of finite Lebesgue measure in $R^{n}$. (Here $R$ denotes the set of reals.) Another proper ideal or $\sigma$-ideal satisfying (4) and (5) comes from the universally measurable sets. Namely, let $S$ be a commutative semitopological group with the topology generated by a complete metric and let $J$ be the ideal or $\sigma$-ideal generated by the family of sets

$\{A \subset S: A$ is universally measurable and $0 \notin$ int $d(A)\}$.

Since, for every $x \in S$, the translations $y \rightarrow x+y, y \in S$, and $y \rightarrow y+x, y \in S$, are homeomorphisms and every homeomorphism maps a universally measurable set onto a universally measurable set, it is easily seen that (4) and (5) are valid. The fact that the ideal or $\sigma$-ideal is proper results from [3, Theorem 1$]$.

\section{REFERENCES}

[1] J. Aczél, J.A. Baker, D.Ž. Djoković, PL. Kannappan and F. Radó, 'Extensions of certain homomorphisms of subsemigroups to homomorphisms of groups', Aequationęs Math. 6 (1971), 263-271.

[2] N.G. de Bruijn, 'On almost additive functions', Colloq. Math. 15 (1966), 59-63.

[3] J.P.R. Christsensen, 'Borel structure in groups and semigroups', Math. Scand. 28 (1971), 124-128.

[4] P. Erdös, 'Problem 310', Colloq. Math. 7 (1960), 311.

[5] P. Fischer and Z. Slodkowski, 'Christensen zero sets and measurable convex functions', Proc. Amer. Math. Soc. 79 (1980), 449-453.

[6] R. Ger, 'Almost additive functions on semigroups and a functional equation', Publ. Math. Debrecen 26 (1979), 219-228.

[7] R. Ger, 'Note on almost additive functions', Aequationes Math. 17 (1978), 73-76.

[8] R. Ger, 'Christensen measurability and functional equations', Grazer Math. Ber. 289 (1988), 1-17.

[9] S. Hartman, 'A remark about Cauchy's equation', Colloq. Math. 8 (1961), 77-79.

[10] W.B. Jurkat, 'On Cauchy's functional equation', Proc. Amer. Math. Soc. 16 (1965), 683-686.

[11] Z. Kominek and M. Kuczma, 'Theorems of Bernstein-Doetsch Piccard and Mehdi and semilinear topology', Arch. Math. (Basel) 52 (1989), 595-602.

[12] K. Stromberg, 'An elementary proof of Steinhaus's theorem', Proc. Amer. Math. Soc. 36 (1972), 308. 
Department of Mathematics Pedagogical University

Rejtana 16 A

35-310 Rzeszów

Poland 